\title{
SOUND TRANSMISSION THROUGH A CYLINDRICAL SANDWICH SHELL WITH HONEYCOMB CORE
}

\author{
Yvette Y. Tang* \\ National Research Council \\ MS 463, NASA Langley Research Center, Hampton, VA 23681 \\ Jay H. Robinson ${ }^{\dagger}$ and Richard J. Silcox ${ }^{\ddagger}$ \\ Structural Acoustic Branch \\ MS 463, NASA Langley Research Center, Hampton, VA 23681
}

\begin{abstract}
Sound transmission through an infinite cylindrical sandwich shell is studied in the context of the transmission of airborne sound into aircraft interiors. The cylindrical shell is immersed in fluid media and excited by an oblique incident plane sound wave. The internal and external fluids are different and there is uniform airflow in the external fluid medium. An explicit expression of transmission loss is derived in terms of modal impedance of the fluids and the shell. The results show the effects of (a) the incident angles of the plane wave; (b) the flight conditions of Mach number and altitude of the aircraft; (c) the ratios between the core thickness and the total thickness of the shell; and (d) the structural loss factors on the transmission loss. Comparisons of the transmission loss are made among different shell constructions and different shell theories.
\end{abstract}

\section{Introduction}

The use of high strength-to-weight ratio composite materials in transport aircraft may result in increased interior noise levels. Previous studies have shown that more noise can be transmitted through laminated fiber-reinforced structures than through isotropic structures in the high frequency range [7]. Noise transmission, measured by transmission loss (TL) through the aircraft, has been studied by Smith [13], White [14], Koval [4 to 7], and Blaise et al. [1] for isotropic, orthotropic, and laminated fiber-reinforced composite shells. Smith presented a theoretical study of transmission of sound energy through a thin, isotropic elastic cylindrical shell from an oblique plane wave excitation. He defined a cross-sectional absorption coefficient that is the ratio of the power absorbed to the incident power per unit length. White investigated sound transmission into an infinite and a finite

* Research Associate, Member AIAA.

$\dagger$ Assistant Branch Head, Member AIAA.

$\ddagger$ Research Engineer, Member AIAA. cylindrical shells and found two important characteristics, the ring and coincidence frequencies, at which TL takes on minima. Koval extended Smith's work to present an analytical model for predicting of TL for isotropic, orthotropic, and laminated fiber-reinforced composite shells. The effect of external airflow was included and the fluids are different inside and outside the shell. The significance of the ring and coincidence frequencies was revealed. Blaise et al. then extended Kaval's work to consider an orthotropic shell excited by an oblique plane sound wave with two independent incident angles. The diffuse field transmission coefficient was examined. Two fluids inside and outside the shell are the same and the external airflow was not included in their study.

The objective of this paper is to study sound transmission through an infinite cylindrical sandwich shell under flight conditions. An aircraft in flight with an external airflow and a negative pressure differential between the inside and outside is modeled as an infinite cylindrical shell. The shell is assumed to be immersed in a fluid media and excited by an incident oblique plane sound wave with two independent angles. The properties of the internal and external fluids surrounding the shell are different. The sandwich shell consists of a honeycomb core and face sheets. The face sheets are made of isotropic materials, orthotropic materials, or laminated fiber-reinforced composite materials. An explicit expression of the frequency spectrum of TL including the effect of the external airflow and two independent incident angles is derived in terms of the modal impedances of the fluids and the shell. In order to study TL, two shell theories are applied. The first is the thin shell theory in which the Reissner-NaghdiBerry theory [10] is used. For thick shells or in the high frequency range of vibrational shells, the transverse shear deformation effect was found to be significant [2, $8,11,12]$. Hence, the first-order shell theory, in which the shear and rotation effects are taken into account, is considered.

The effects of different source conditions, structural properties and flight conditions on TL are studied for a range of values, specifically, incident angle of the plane wave; Mach number and the flight altitude of the aircraft; the ratios between the core thickness and 
the total thickness of the shell, and the structural loss factors are shown. Comparisons of TL are made among an aluminum monocoque shell, an aluminum sandwich shell with aluminum face sheets and an aluminum core, and a composite sandwich shell with laminated graphite/epoxy face sheets and the same aluminum core as aluminum sandwich shell and between a thin shell theory and the first-order shell theory.

\section{Mathematical model}

Figure 1 shows an infinite cylindrical sandwich shell with radius $R$ and wall thickness $h$. The shell is immersed in fluid medium 1 (external fluid) and contains fluid medium 2 (internal fluid). The mass density and sound speed for fluid media 1 and 2 are $\left\{\rho_{1}\right.$, $\left.c_{1}\right\}$ and $\left\{\rho_{2}, c_{2}\right\}$, respectively. An oblique plane sound wave $p^{l}$ is approaching the shell with incident angles $\left\{\gamma_{1}, \psi_{1}\right\}$. Airflow in fluid medium 1 (external) is moving with a constant velocity $\mathbf{V}=\left\{V_{x}, V_{y}, V_{z}\right\}$ in the $x, y$, and $z$ directions.

\subsection{Governing equations of the fluids}

Due to the existence of airflow in the external fluid medium, the external pressure, which is the summation of the incident wave $p^{l}$ and the reflected wave $p^{R}$, satisfies the convected wave equation

$$
c_{1}^{2} \nabla^{2}\left(p^{I}+p^{R}\right)+\left(\frac{\partial}{\partial t}+\mathbf{V} \cdot \nabla\right)^{2}\left(p^{I}+p^{R}\right)=0
$$

where $\nabla$ and $\nabla^{2}$ are the gradient operator and the Laplacian operator, respectively. equation

The internal pressure satisfies the acoustic wave

$$
c_{2}^{2} \nabla^{2} p^{T}+\frac{\partial^{2} p^{T}}{\partial t^{2}}=0
$$

where $p^{T}$ is the transmitted wave. Note that Eq. (2) implies that the shell interior is totally absorptive. Therefore only an inward-traveling wave exists in the shell interior. Although this is not representative of an aircraft interior, the model allows the transmission loss of the cylindrical wall to be studied in a straight forward manner.

\subsection{Governing equations of the sandwich shell}

Let the non-dimensional axial coordinate be $\xi$ $=z / R$, the circumferential direction be $\theta$, and the normal direction to the middle surface of the shell be $\zeta$, as shown in Fig. 2. Denote the shell displacement components at the midsurface in the axial, circumferential, and radial directions as $\left\{u^{0}, v^{0}, w^{0}\right\}$, respectively. Let $\left\{\psi_{\xi}, \psi_{\theta}\right\}$ be the rotations of the normal direction relative to the undeformed midsurface. The displacement components at an arbitrary distance $\zeta$ from the midsurface along the axial, the circumferential, and the radial directions are

$$
u=u^{0}+\zeta \psi \xi, v=v^{0}+\zeta \psi_{\theta}, w=w^{0}
$$

It should be mentioned that if the thin shell theory is applied, then the rotations are $\psi \xi=w, \xi / R$ and $\psi_{\theta}=w{ }_{\theta}^{0} / R$, where the comma denotes the partial derivative with respect to the named variable.

Using the strain-displacement relations, the constitutive laws, and the equations of motion of the three-dimensional theory of elasticity in the cylindrical coordinate system $\{r, \theta, \xi\}$, where $r$ is the radial coordinate, one can obtain the following equations of motion for an $N$-layered cylindrical sandwich shell [2]

$$
\begin{aligned}
& N_{1, \xi}+N_{6, \theta}=R_{0} R u_{, t t}^{0}+R_{1} R \psi \psi_{, t t} \\
& N_{2, \theta}+N_{6, \xi}-Q_{2}=R_{0} R v_{, t t}^{0}+R_{1} R \psi_{\theta, t t} \\
& Q_{1, \xi}+Q_{2, \theta}+N_{2}=R_{0} R w_{, t t}^{0}+\left.\left[p^{T}-\left(p^{I}+p^{R}\right)\right]\right|_{r=R} \\
& M_{1, \xi}+M_{6, \theta}-R Q_{1}=R_{1} R u_{, t t}^{0}+R_{2} R \psi_{\xi, t t} \\
& M_{2, \theta}+M_{6, \xi}-R Q_{2}=R_{1} R v_{, t t}^{0}+R_{2} R \psi_{\theta, t t}
\end{aligned}
$$

where $t$ is time. Equations (4) to (6) present the equation of the translation motion and Eqs. (7) and (8) present the equations of rotational motion. The meaning of each term in these equations is explained in the following.

The inertia terms $R_{0}, R_{1}$, and $R_{2}$ in Eqs. (4) to (8) are defined by

$$
\left\{R_{0}, R_{1}, R_{2}\right\}=\sum_{k=1}^{N} \int_{h k} \rho^{k}\left\{1, \zeta, \zeta^{2}\right\} d \zeta
$$

where $\rho^{k}$ and $h_{k}$ are the mass density and the thickness of the $k$-th layer.

In Eqs. (4) to (8), the stress resultants $N_{1}, N_{2}$, and $N_{6}$ and couples $M_{1}, M_{2}$, and $M_{6}$ are given by

$$
N_{i}=A_{i j} \varepsilon_{j}^{0}+B_{i j} \chi_{j}^{0}, \quad M_{i}=B_{i j} \varepsilon_{j}^{0}+D_{i j} \chi_{j}^{0}(10)
$$

where the subscripts $(i, j)(1,2,6)$ follow Voigt's notations and the stretching, bending-stretching coupling, and bending stiffness constants $A_{i j}, B_{i j}$, and $D_{i j}$ are

$$
\left\{A_{i j}, B_{i j}, D_{i j}\right\}=\sum_{k=1}^{N} \int_{h k} c_{i j}^{k}\left\{1, \zeta, \zeta^{2}\right\} d \zeta
$$

and $c_{i j}^{k}$ are the transformed reduced stiffness constants of the $k$-th layer [3]. The strains at the midsurface $\varepsilon_{i}^{0}$ in Eq. (10) are

$$
\left\{\varepsilon_{1}^{0}, \varepsilon_{2}^{0}, \varepsilon_{6}^{0}\right\}=\left\{u, \xi,\left(v_{, \theta}^{0}-w^{0}\right),\left(v, \xi+u_{, \theta}^{0}\right)\right\} / R(12)
$$

and the curvatures at the midsurface $\chi_{i}^{0}$ for the thin shell theory are

$$
\left\{\chi_{1}, \chi_{2}, \chi_{6}\right\}=\left\{w_{, \xi \xi}^{0},\left(w_{, \theta \theta}^{0}-v_{, \theta}^{0}\right),\left(2 w_{, \xi}^{0}-v_{, \xi}^{0}\right)\right\} / R^{2}
$$

and for the first-order shell theory are

$$
\left\{\chi_{1}, \chi_{2}, \chi_{6}\right\}=\left\{\psi \xi, \xi, \psi_{\theta, \theta},\left(\psi_{\xi, \theta}+\psi_{\theta, \xi}\right)\right\} / R(14)
$$

The transverse shear forces $Q_{1}$ and $Q_{2}$ in Eqs. (5) to (8) are obtained differently for the thin shell theory and the first-order shell theory. For the thin shell theory, because the effects of shear and rotation are neglected, the inertial terms $R_{1}=R_{2}=0$ and the shear forces $Q_{1}$ and $Q_{2}$ can be obtained directly from Eqs. (7) 
and (8). However, for the first-order shell theory, the shear forces $Q_{1}$ and $Q_{2}$ are

$Q_{1}=k_{4}^{2} A_{44}\left(w_{, \xi}^{0} / R+\psi_{\xi}\right)+k_{4} k_{5} A_{45}\left(w_{, \theta}^{0} / R+\psi_{\theta}\right)$

$Q_{2}=k_{5}^{2} A_{55}\left(w_{, \theta}^{0} / R+\psi_{\theta}\right)+k_{4} k_{5} A_{45}(w, \xi / R+\psi \xi)$

Here, in Eqs. (15) and (16), $k_{4}$ and $k_{5}$ are the shear correction factors and the shear elastic constants $A_{44}$, $A_{45}$, and $A_{55}$ are

$\left\{A_{44}, A_{45}, A_{55}\right\}=\sum_{k=1}^{N} \int_{h_{k}}\left\{c_{44}^{k}, c_{45}^{k}, c_{55}^{k}\right\} d \zeta$

where $c_{44}^{k}, c_{45}^{k}$, and $c_{55}^{k}$ are the transformed shear stiffness constants of the $k$-th layer [3].

\subsection{Boundary conditions}

The boundary conditions inside and outside the shell are given by following

$$
\begin{aligned}
& \left.\frac{\partial\left(p^{I}+p^{R}\right)}{\partial r}\right|_{r=R}=-\rho_{1}\left(\frac{\partial}{\partial t}+\mathbf{V} \cdot \nabla\right)^{2} w \\
& \left.\frac{\partial p^{T}}{\partial r}\right|_{r=R}=-\rho_{2} \frac{\partial^{2} w}{\partial t^{2}}
\end{aligned}
$$

which describe the effect of the fluid pressure on the shell motion.

\subsection{Solutions}

To obtain the solution of Eq. (1), the given incident wave $p^{l}$ can be expanded as

$p^{I}=p_{0} \sum_{n=0}^{\infty} \varepsilon_{n}(-\mathrm{i})^{n} J_{n} \cos \left[n\left(\theta-\psi_{1}\right)\right] \exp \left[\mathrm{i}\left(\omega t-k_{1 z} z\right)\right]$ (20)

where $p_{0}$ is the amplitude of the incident wave, $n$ the order of the circumferential mode, $\varepsilon_{n}=1$ when $n=0$ and $\varepsilon_{n}=2$ when $n \neq 0$, and $J_{n}=J_{n}\left(k_{1} r\right)$ the Bessel function of the first kind of order $n$. The wave numbers $k_{1 r}$ and $k_{1 z}$ are to be determined. The reflected wave $p^{R}$ can be obtained by following

$p^{R}=\sum_{n=0}^{\infty} A_{n} H_{n}^{2} \cos \left[n\left(\theta-\psi_{1}\right)\right] \exp \left[\mathrm{i}\left(\omega t-k_{1 z} z\right)\right]$

where $H_{n}^{2}=H_{n}^{2}\left(k_{1} r\right)$ is the Hankel function of the second kind of order $n$ and $A_{n}$ are yet-to-be-determined complex amplitude factors. Substituting Eqs. (20) and (21) into Eq. (1), one can obtain the wave numbers

$k_{1 z}=k_{1} \cos \gamma_{1}, k_{1 r}=k_{1} \sin \gamma_{1}$

where

$k_{1}=\frac{\omega}{c_{1}} \times$

$1-\left(M_{x} \sin \gamma_{1} \cos \psi_{1}+M_{y} \sin \gamma_{1} \sin \psi_{1}+M_{z} \cos \gamma_{1}\right)$

$M_{x}=V_{x} / c_{1}, M_{y}=V_{y} / c_{1}, M_{z}=V_{z} / c_{1}$

The transmitted wave $p^{T}$, which satisfies Eq. (2), is

$p^{T}=\sum_{n=0}^{\infty} B_{n} H_{n}^{1} \cos \left[n\left(\theta-\psi_{1}\right)\right] \exp \left[\mathrm{i}\left(\omega t-k_{2 z} z\right)\right]$

where $H_{n}^{1}=H_{n}^{1}\left(k_{2} r r\right)$ is the Hankel function of the first kind of order $n$, $k_{2}=\omega / c_{2}, k_{2 z}=k_{1 z}, k_{2 r}=\sqrt{k_{2}^{2}-k_{2 z}^{2}}$

and $B_{n}$ are yet-to-be-determined complex amplitude factors.

The displacement components $u^{0}, v^{0}$, and $w^{0}$ are also assumed as

$$
\begin{aligned}
& u^{0}=\sum_{n=0}^{\infty} \mathrm{i} u_{n}^{0} \cos \left[n\left(\theta-\psi_{1}\right)\right] \exp \left[\left(\omega t-k_{1 z} z\right)\right] \\
& v^{0}=\sum_{n=0}^{\infty} v_{n}^{0} \sin \left[n\left(\theta-\psi_{1}\right)\right] \exp \left[\left(\omega t-k_{1 z} z\right)\right] \\
& w^{0}=\sum_{n=0}^{\infty} w_{n}^{0} \cos \left[n\left(\theta-\psi_{1}\right)\right] \exp \left[\left(\omega t-k_{1 z} z\right)\right]
\end{aligned}
$$

For the first-order shell theory, the rotation components $\psi \xi$ and $\psi_{\theta}$ are assumed as

$$
\begin{aligned}
& \psi_{\xi}=\sum_{n=0}^{\infty} \mathrm{i} u_{n}^{1} \cos \left[n\left(\theta-\psi_{1}\right)\right] \exp \left[\left(\omega t-k_{1 z} z\right)\right] \\
& \psi_{\theta}=\sum_{n=0}^{\infty} v_{n}^{1} \sin \left[n\left(\theta-\psi_{1}\right)\right] \exp \left[\left(\omega t-k_{1 z} z\right)\right]
\end{aligned}
$$

Here, in Eqs. (27) to (31), $\left\{u_{n}^{0}, v_{n}^{0}, w_{n}^{0}\right\}$ and $\left\{u_{n}^{1}, v_{n}^{1}\right\}$ are unknown complex amplitudes of the displacement and rotation components, respectively. It can be seen that for each mode number $n$, there are five unknowns $\left\{u_{n}^{0}, v_{n}^{0}, w_{n}^{0}, A_{n}, B_{n}\right\}$ and five equations (4) to (6), (18), and (19) for the thin shell theory and seven unknowns $\left\{u_{n}^{0}, v_{n}^{0}, w_{n}^{0}, u_{n}^{1}, v_{n}^{1}, A_{n}, B_{n}\right\}$ and seven equations (4) to (8), (18), and (19) for the first-order shell theory. To obtain simplified and meaningful results, one can get a set of equations in terms of $A_{n} \quad B_{n}$ and $w_{n}^{0}$ by eliminating $u_{n}^{0}$ and $v_{n}^{0}$ for the thin shell theory and $u_{n}^{0}$, $v_{n}^{0}, u_{n}^{1}$, and $v_{n}^{1}$ for the first-order shell theory. Solving these coupled equations yields

$$
\begin{aligned}
& A_{n}=-p_{0} \varepsilon_{n}(-\mathrm{i})^{n} \frac{J_{n}^{\prime}\left(k_{1 r} R\right)}{H_{n}^{2^{\prime}}\left(k_{1} R\right)} \frac{\left(Z_{n}^{S}+Z_{n}^{T}+Z_{n}^{I}\right)}{\left(Z_{n}^{S}+Z_{n}^{T}+Z_{n}^{R}\right)} \\
& B_{n}=p_{0} \varepsilon_{n}(-\mathrm{i})^{n} \frac{\rho_{2} k_{1} J_{n}^{\prime}\left(k_{1 r} R\right) Z_{n}^{R}+Z_{n}^{I}\left(k_{1} c_{1} / \omega\right)^{2}}{\rho_{1} k_{2} H_{n}^{1^{\prime}}\left(k_{2 r} R\right) \quad Z_{n}^{S}+Z_{n}^{T}+Z_{n}^{R}} \\
& w_{n}^{0}=p_{0} \varepsilon_{n}(-\mathrm{i})^{n} \frac{k_{1} J_{n}\left(k_{1} R\right) Z_{n}^{R}+Z_{n}^{I}\left(k_{1} c_{1} / \omega\right)^{2}}{\rho_{1} \omega^{2} \quad Z_{n}^{S}+Z_{n}^{T}+Z_{n}^{R}}
\end{aligned}
$$

where

$$
\begin{aligned}
& Z_{n}^{I}=\mathrm{i} \omega \frac{\rho_{1}}{k_{1 r}} \frac{J_{n}\left(k_{1} R\right)}{J_{n}^{\prime}\left(k_{1} R\right)} \\
& Z_{n}^{R}=-\mathrm{i} \omega \frac{\rho_{1}}{k_{1} r} \frac{H_{n}^{2}\left(k_{1 r} R\right)}{H_{n}^{2^{\prime}}\left(k_{1 r} R\right)}\left(\frac{k_{1} c_{1}}{\omega}\right)^{2} \\
& Z_{n}^{T}=\mathrm{i} \omega \frac{\rho_{2}}{k_{2 r}} \frac{H_{n}^{1}\left(k_{2} R\right)}{H_{n}^{1^{\prime}}\left(k_{2} R\right)} \\
& Z_{n}^{S}=\frac{1}{\mathrm{i} \omega D_{n}}
\end{aligned}
$$

Here, in Eqs. (32) to (38), the primes denote derivatives with respect to the argument; $Z_{n}^{I}, Z_{n}^{R}$, and $Z_{n}^{T}$ are modal characteristic acoustic impedances of the fluids; $Z_{n}^{S}$ is the modal impedance of the shell; and $D_{n}$ is the modal amplitude of the displacement component of the shell in the radial direction with a unit pressure. 


\subsection{Transmission loss}

The transmission loss of the shell is defined by the ratio of the transmitted power $W^{T}$ and the incident power $W^{l}$ per unit length of axial section of the shell as follows [9]

where

$$
T L=-10 \log _{10} \frac{W^{T}}{W^{I}}
$$

$$
\begin{aligned}
W^{I} & =\frac{p_{0}^{2} R \cos \gamma_{1}}{\rho_{1} c_{1}} \\
W^{T} & =\left.\frac{1}{2} \operatorname{Re} \int_{S}\left(p^{T} \dot{w}^{*}\right)\right|_{r=R} d S
\end{aligned}
$$

in which $\mathrm{Re}[$.$] is the real part, the superscript *$ represents the complex conjugate of the argument, and $S$ is the area of the cross section of the shell. Substituting Eqs. (25) and (29) by using Eqs. (33) and (34) into Eq. (39) leads to the expression of TL in terms of the modal impedances

$$
\begin{gathered}
T L=-10 \log _{10} \sum_{n=0}^{\infty} \frac{2 \varepsilon_{n}}{k_{1 r} R}\left(\frac{\omega}{k_{1} c_{1}}\right)^{2} * \\
\frac{\operatorname{Re}\left[Z_{n}^{R}\right] \operatorname{Re}\left[Z_{n}^{T}\right]}{\left|Z_{n}^{R}+Z_{n}^{T}+Z_{n}^{S}\right|^{2}}
\end{gathered}
$$

where |.| is the absolute value of the argument.

\section{Parametric studies}

Parametric numerical studies of transmission loss (TL) are conducted for frequency from 0.1 to 20 times the ring frequency $f_{R}$. These studies provide insight into the effect of the acoustic properties of the fluids and the structural or material parameters of the shells on TL. A typical narrow-bodied jet fuselage made of the sandwich shells with radius $R=1.83 \mathrm{~m}$ is considered. The inner and outer face sheets are assumed to be the same. The properties of the fluids and the shells are as follows:

The aircraft is in cruising flight at $10,000 \mathrm{ft}\left(\rho_{1}=\right.$ $\left.0.9041 \mathrm{~kg} / \mathrm{m}^{3}, \quad c_{1}=328.558 \mathrm{~m} / \mathrm{s}\right), 25,000 \mathrm{ft}\left(\rho_{1}=\right.$ $\left.0.5489 \mathrm{~kg} / \mathrm{m}^{3}, c_{1}=309.966 \mathrm{~m} / \mathrm{s}\right)$, and $35,000 \mathrm{ft}\left(\rho_{1}=\right.$ $0.3795 \mathrm{~kg} / \mathrm{m}^{3}, c_{1}=296.556 \mathrm{~m} / \mathrm{s}$ ) with Mach number $M_{x}=M_{y}=0$, and $M_{z}=0,0.5,1.0,1.5$. The fuselage interior is pressurized to $10,000 \mathrm{ft}$, such that no pressure differential exists at $10,000 \mathrm{ft}$. The plane sound wave approaches the shell with incident angles $\gamma_{1}=150^{\circ}$, $135^{\circ}$, and $120^{\circ}$ and $\psi_{1}=0^{\circ}$, respectively.

Two kinds of the sandwich shells with the same weight, radius, and aluminum core are considered. One is an aluminum sandwich shell whose face sheets are made of aluminum, and the other is a composite sandwich shell whose face sheets are made of laminated cross-ply graphite/epoxy layers. The core has low density and high out-of-plane modulus. Therefore, assume the material constants of the aluminum core including the mass density, Young's modulus, Poison's ratio, and shear modulus to be $\rho=55 \mathrm{~kg} / \mathrm{m}^{3}, E \approx 0, \mu \approx$ 0 , and $G=1.38 \mathrm{GPa}$, respectively. The core thickness of both aluminum and composite sandwich shells is denoted as $h_{c}$. The material constants of the aluminum face sheets are $\rho=2750 \mathrm{~kg} / \mathrm{m}^{3}, E=72 \mathrm{GPa}$, and $\mu=$ 0.3. The material constants of the laminated graphite/epoxy face sheets are $\rho=1600 \mathrm{~kg} / \mathrm{m}^{3}, E_{\alpha}=$ $221 \mathrm{GPa}, E_{\beta}=6.9 \mathrm{GPa}, \mu_{\alpha \beta}=0.25$, and $G_{\alpha \beta}=4.8 \mathrm{GPa}$, where $\alpha$ is the fiber direction and $\beta$ is the direction perpendicular to the fiber. Both inner and outer laminated graphite/epoxy face sheets consist of four layers with the fiber orientations $\left\{90^{\circ}, 0^{\circ}, 90^{\circ}, 0^{\circ}\right\}$ and $\left\{0^{0}, 90^{\circ}, 0^{0}, 90^{\circ}\right\}$ with $\xi$ direction on the shell outer surface, respectively. The ratio of the core thickness and the total thickness of the shell is $h_{c} / h=0.8,0.9$, and 0.95. The structural loss factors are assumed as $\eta=$ $0.01,0.05$, and 0.1 .

For reference purposes, the same weight and radius aluminum monocoque shell with the wall thickness $h=$ $0.159 \mathrm{~cm}$ is considered (other material constants are given above). Then the wall thicknesses of the aluminum and composite sandwich shells are $h=$ $0.736 \mathrm{~cm}$ and $h=1.2 \mathrm{~cm}$, respectively. The frequency parameter of all results in the following figures is normalized by the ring frequency of the aluminum monocoque shell, $f_{R}=(2 \pi R)^{-1} \sqrt{E / \rho}=445 \mathrm{~Hz}$. The effect of the pressure on TL is neglected since contribution of the pressure to the shell mode impedance is less than $1 \%$.

\section{Results}

The effects of the incident angles of the plane sound wave, the Mach numbers, the flight altitudes, the ratios of the core thickness to the total thickness of the shell, and the loss factors of the shell material on TL are shown in Figs. 3 through 7 based on the thin shell theory. Figures (a) and (b) represent TL versus the normalized frequency for the aluminum sandwich shell and the composite sandwich shell, respectively.

\subsection{The effect of the fluid properties on TL}

Figure 3 shows the effect of the incident angles with $\gamma_{1}=150^{\circ}, 135^{\circ}$, and $120^{\circ}$ on TL. There are two major minima in TL for the aluminum sandwich shell at the ring frequency $f_{R}$ and the critical frequency $f_{c}$, $\left(>f_{R}\right)$ which corresponds to the coincidence frequency of an infinite plate with the same shell thickness, as shown in Fig. 3(a). The frequency $f_{c}$ is shifted upwards when the plane sound wave is close to the normal incidence $\left(\gamma_{1}=90^{\circ}\right)$ (see Fig. 3(a)). However, only one major minimum in TL at $f_{R}$ is observed for the composite sandwich shell, as shown in Fig. 3(b). In this case, the bending wave of the shell that corresponds to $f_{c}$ becomes unimportant for sound transmission in the composite sandwich shell when $f_{c}<f_{R}$.

Figure 4 shows the effect of Mach numbers with $M_{z}=0,0.5,1.0$, and 1.5 on TL. Two major minima of TL at $f_{R}$ and $f_{c}$ occur for the aluminum sandwich 
shell. Only one major minimum in TL at $f_{c}$ is seen in the composite sandwich shell, as shown in Fig. 4(b).

The frequency $f_{c}$ is shifted upwards with increase of Mach number for both sandwich shells.

Figure 5 shows the effect on TL of altitudes of $10,000 \mathrm{ft}, 25,000 \mathrm{ft}$, and 35,000ft altitudes with the same interior pressure at $10,000 \mathrm{ft}$. The effect of the pressure differential inside and outside the shell on the model impedance of the shell is small so that it can be neglected. A higher flight altitude will lead to a larger acoustic impedance $\left(c_{i} \rho_{i}\right)$ mismatch between the fluids inside and outside the shell. The curves in Figs. 5a and $5 \mathrm{~b}$ illustrate that with an increase of the acoustic impedance mismatch, the transmission loss increases except near $f_{c}$ (see Fig. 5). Figure 5(a) shows that $f_{c}$ is shifted downwards slightly when the flight altitude increases. Figure 5(b) shows only one major minimum in TL at $f_{R}$ for the composite sandwich shell.

\subsection{The effect of the structural properties on TL}

Figure 6 shows the effect of the different thickness ratios with $h_{c} / h=0.8,0.9$, and 0.95 on TL. For the ratio $h_{c} / h$ increasing, this indicates that the core thickness increases while the thicknesses of the face sheets decrease since the total thickness is kept constant for each shell. The increase of $h_{c} / h$ will results in that the bending stiffness is improved and the in-lane stiffness is the same since bending modes are more important at higher frequencies to transmit wave. The minimum in TL at $f_{c}$ is observed only when $h_{c} / h=0.8$ for the aluminum sandwich shell, as shown in Fig. 6(a). The curves in Fig. 6 reveal that when $f<f_{R}$ the transmission loss follows a stiffness-controlled law in which TL decreases 5-6 dB/octave and when $f>f_{c}$ for the aluminum sandwich shell and $f>f_{R}$ for the composite sandwich shell, the transmission loss increases about $20 \mathrm{~dB}$ /octave.

Figure 7 shows the effect of the structural loss factors with $\eta=0.01,0.05$, and 0.1 on TL. The structural damping becomes unimportant in the study of TL except at frequencies $f_{c}$ and $f_{R}$ where magnitude of TL increases with increase of the loss factor near $f_{c}$ and $f_{R}$ as shown in Fig. 7(a).

\subsection{Comparison of TL among different shells}

Figures 8(a) and (b) show TL of the aluminum monocoque shell, aluminum sandwich shell, and composite sandwich shell with $h_{c} h=0.8$ and $h_{c} h=$ 0.95 , respectively. Below $f_{R}$, transmission loss is almost the same in the aluminum monocoque shell and in the aluminum sandwich shell. It can be seen that when $f>f_{R}$, the transmission loss of the composite sandwich shell is the largest among the shells. However, when $f<f_{R}$, the transmission loss for the composite sandwich shell is the least of the three shells. It can also be seen that at high frequencies, an increase in wall thickness ratio $\left(h_{c} / h\right)$ has a large effect on TL for the sandwich shell than the monocoque shell.

Figures 9(a) and (b) show TL the aluminum monocoque shell, aluminum sandwich shell, and composite sandwich shell at altitudes 25,000ft and $35,000 \mathrm{ft}$ with the same interior pressure (at 10,000 ft altitude), respectively. Below $f_{R}$, transmission loss is almost the same in the aluminum monocoque shell and in the aluminum sandwich shell. Results illustrate that the composite sandwich shell offers an advantage in noise reduction over the aluminum monocoque and sandwich shells in the high frequency range.

\subsection{Comparison of TL for different shell theories}

Figures 10(a) and (b) show a comparison on TL for the thin shell theory and the first-order shell theory. The selected shear correction factors $k_{4}=k_{5}=\sqrt{ } 5 / 6$. At the lower frequency, the effects of shear and rotation on TL are negligible. However, in the high frequency range, the shear waves transmit sound through the shell resulting in a decrease of TL. The effect of the shear wave on sound transmission becomes more important for the composite sandwich shell than the aluminum sandwich shell because the composite sandwich shell is thicker than the other.

\section{Conclusion}

In this paper, we studied sound transmission through an infinite cylindrical sandwich shell. An explicit expression for the shell transmission loss (TL) including the effect of the external airflow and two independent incident angles is derived. The frequency spectrum of TL versus the normalized frequency is studied by varying parameters of the fluids and shells. Comparison of TL is made among the aluminum monocoque shell, aluminum sandwich shell, and composite sandwich shell based on the thin shell theory and between the thin and first order shell theories, respectively. The following conclusions can be drawn from this numerical study:

1. Two major minima in TL at $f_{R}$ and $f_{c}$ are present in the aluminum sandwich shell. However, only one major minimum in TL at $f_{R}$ is shown in the composite sandwich shell. In this case, the bending wave of the composite sandwich shell becomes unimportant for sound transmission because no major minimum in TL is shown at $f_{c}$.

2. Increase of the ratio $h_{c} / h$ will lead to an increase of $\mathrm{TL}$ in the high frequency range. The structural damping is unimportant in the study of TL although the magnitude of TL varies slightly for different dampings near at $f_{R}$ and $f_{c}$.

3. The composite sandwich shell offers an advantage in noise reduction over both the aluminum monocoque shell and the aluminum sandwich shell for the larger impedance mismatch between the fluids inside and outside the shell and the larger 
ratio of the core thickness and total thickness in high frequency range based on thin shell theory at the high frequency.

4. The effects of shear and rotation of the sandwich shells on TL can be neglected only in the lower frequency range. In the high frequency range, the shear wave's transmission of sound will result in decreased TL.

\section{References}

1. Blaise, A., Lesueur, C., Gotteland, M., and Barbe, M., "On sound transmission into an orthotropic infinite shell: Comparison with Koval's results and understanding of phenomena", Journal of Sound and Vibration, Vol. 150, No. 2, 1991, pp. 233-243.

2. Greenberg, J. B. and Stavsky, Y., "Vibration of axially compressed laminated orthotropic cylindrical shells, including transverse shear deformation", Acta Mechanica, Vol. 37, 1980, pp. 13-28.

3. Jones, R. M., "Mechanics of composite materials", Hemisphere publishing corporation", New York, 1975, pp. 51.

4. Koval, L. R., "On sound transmission into a thin cylindrical shell under 'flight conditions'", Journal of Sound and Vibration, Vol. 48, No. 2, 1975, pp. 265-275.

5. Koval, L. R., "Effect of stiffening on sound transmission into a cylindrical shell in flight", AIAA Journal , Vol. 15, No. 7, 1977, pp. 899900.

6. Koval, L. R., "On Sound transmission into an orthotropic shell", Journal of Sound and Vibration, Vol. 63, No. 1, 1979, pp. 51-59.

7. Koval, L. R., "Sound transmission into a laminated composite cylindrical shell", Journal of Sound and Vibration, Vol. 71, No. 4, 1980, pp. 523-530.

8. Leissa, A. W., "Vibration of shells", NASA SP288, 1973, pp. 291-298.

9. Lesueur, C., "Rayonnement acoustique des structures-vibroacouctique-interactions fluidestructure", Paris: Collection EDF-DER, Editions Eyrolles, 1988, pp. 231-239.

10. Markus, S., "The mechanics of vibrations of cylindrical shells", Elsevier, 1988, pp. 64-65.

11. Mindlin, R. D., "Influence of rotatory inertia and shear on flexural motions of isotropic elastic plates", J. Mech. , Vol. 18, No. 1, 1951, pp. 3138.

12. Reissner, E., "The effect of transverse shear deformation on the bending of elastic plate", $J$. Appl. Mech. , Vol. 12, 1945, A69-A77.

13. Smith, P. W., Jr., "Sound transmission through thin cylindrical shells", Journal of the Acoustical Society of America, Vol. 29, No. 6, 1957, pp. 721-729.
14. White, P. "Sound transmission through an finite, closed, cylindrical shell", Journal of the Acoustical Society of America, Vol. 40, No. 5., 1966, pp. 1124-1130.

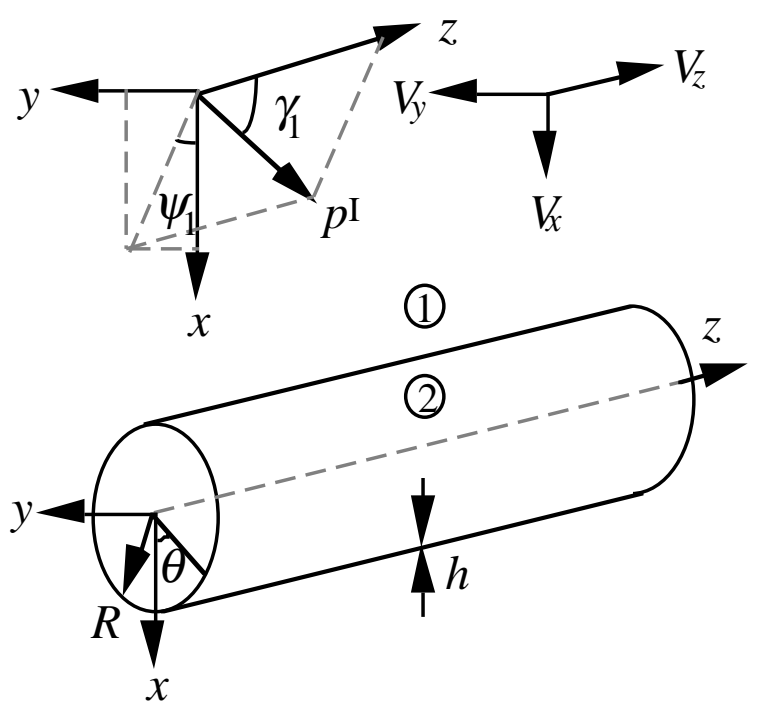

Figure 1. System configuration.

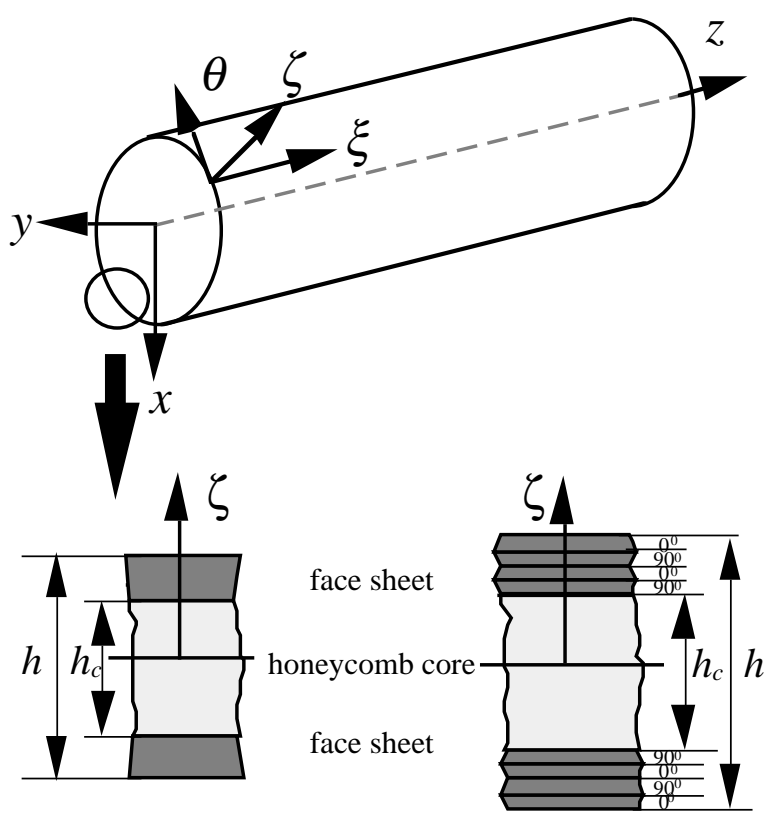

aluminum sandwich

composite sandwich

Figure 2. Geometry of the sandwich shells. 

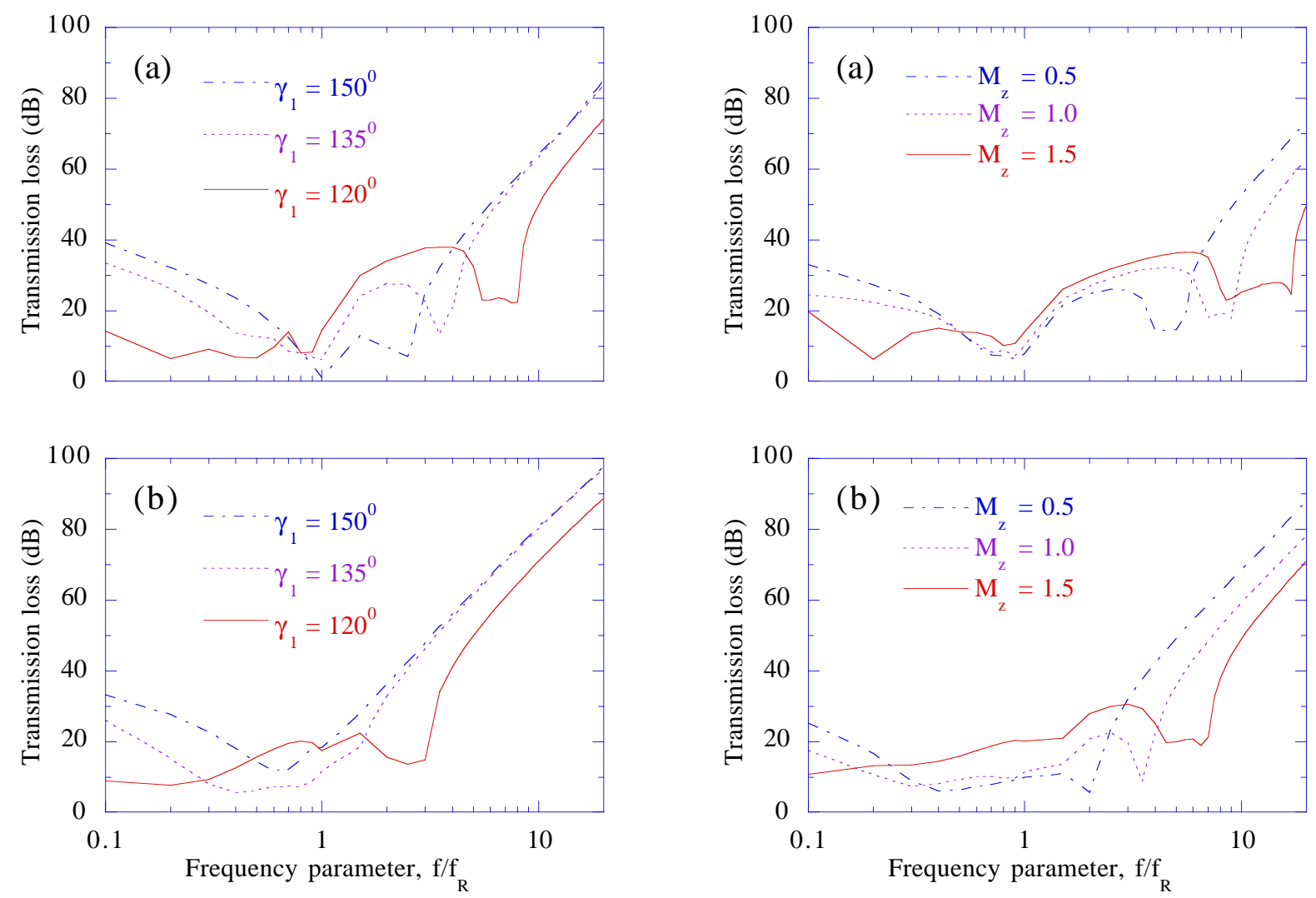

Figure 3. The effect of the incident angles on the TL at no airflow with the flight altitude $(25,000 \mathrm{ft})$ and the interior pressure $(10,000 \mathrm{ft}): \quad \eta=0.01 ; h_{c} / h=0.8$. (a). aluminum sandwich shell; (b) composite sandwich shell.

Figure 4. The effect of the Mach numbers on the TL with the flight altitude $(25,000 \mathrm{ft})$ and the interior pressure $(10,000 \mathrm{ft}): \quad \eta=0.01 ; \gamma_{1}=150^{\circ} ; h_{c} / h=0.8$. (a). aluminum sandwich shell; (b) composite sandwich shell. 

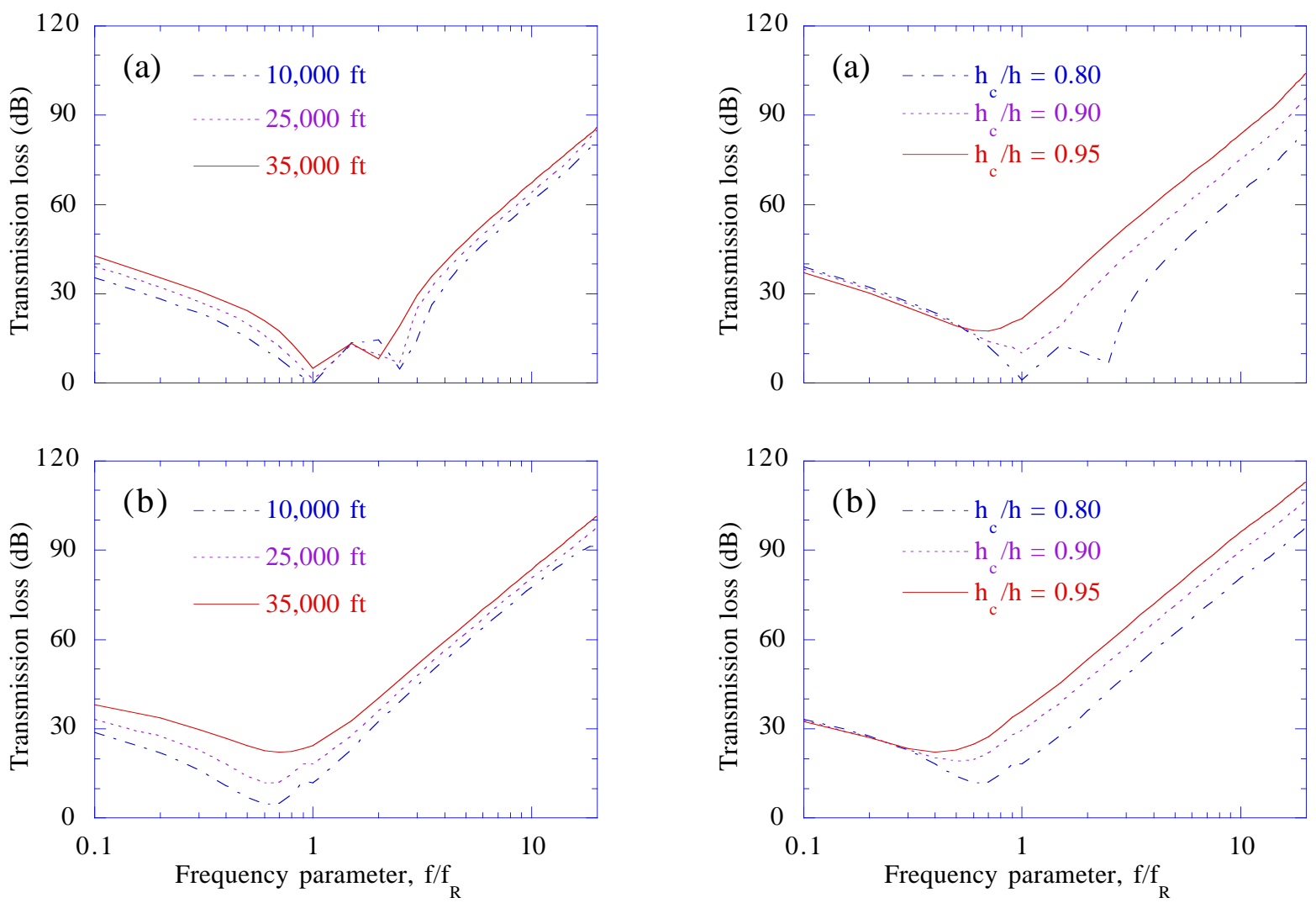

Figure 5. The effect of the flight altitudes on the TL at no airflow with the interior pressure $(10,000 \mathrm{ft})$ : $\eta=$ $0.01 ; \gamma_{1}=150^{\circ} ; h_{c} / h=0.8$. (a). aluminum sandwich shell; (b) composite sandwich shell.

Figure 6. The effect of the thickness on the TL ratios at no airflow with the flight altitude $(25,000 \mathrm{ft})$ and the interior pressure $(10,000 \mathrm{ft}): \quad \eta=0.01 ; \gamma_{1}=150^{\circ}$. (a). aluminum sandwich shell; (b) composite sandwich shell. 

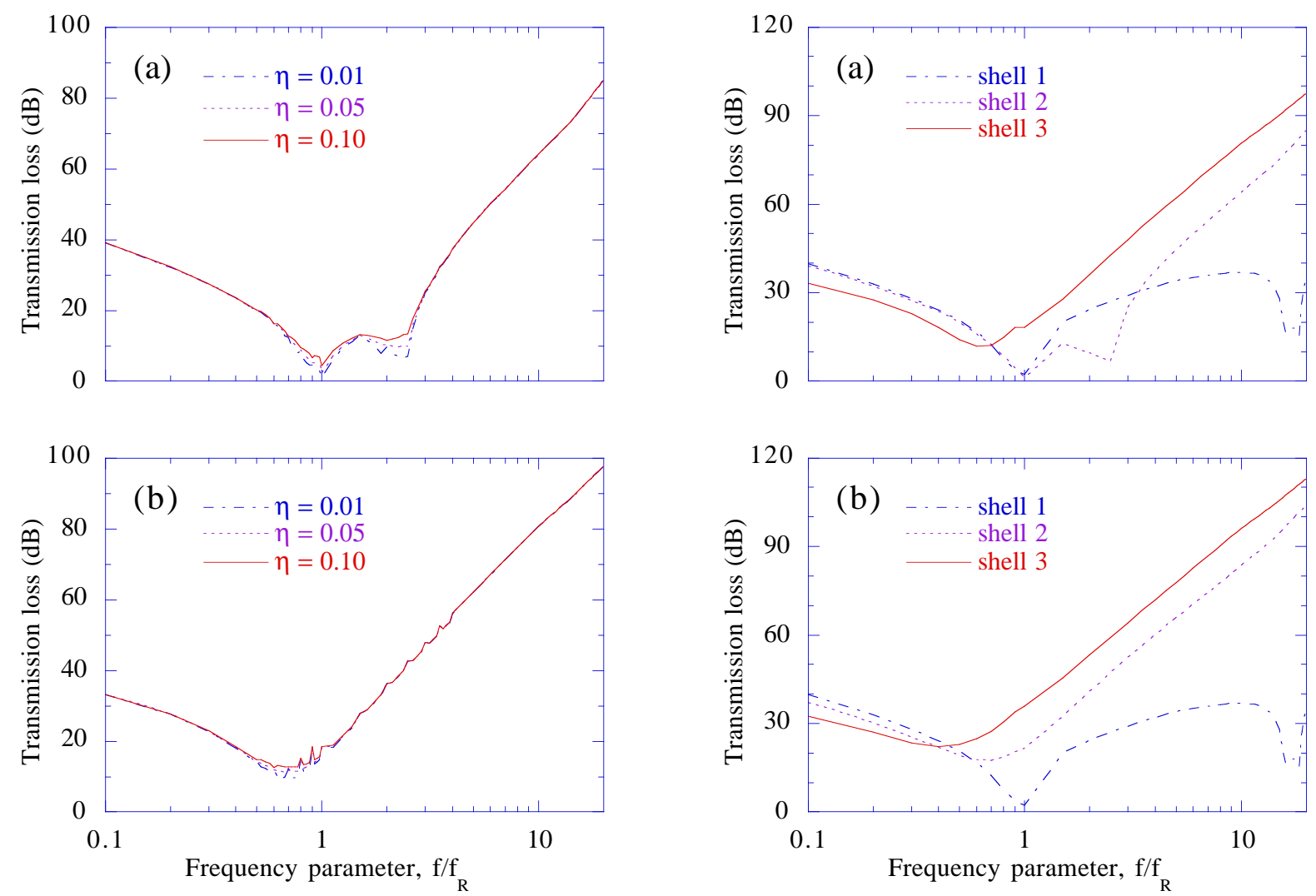

Figure 7. The effect of the structural loss factors on the TL at no airflow with the flight altitude $(25,000 \mathrm{ft})$ and the interior pressure $(10,000 \mathrm{ft}): \gamma_{1}=150^{\circ} ; h_{c} / h=0.8$. (a). aluminum sandwich shell; (b) composite sandwich shell.

Figure 8. Comparison of the effect of the thickness ratio on the TL at no airflow with the flight altitude $(25,000 \mathrm{ft})$ and the interior pressure $(10,000 \mathrm{ft}): \quad \eta=$ $0.01 ; \gamma_{1}=150^{\circ}$. shell 1: aluminum monocoque shell; shell 2: aluminum sandwich shell; shell 3: composite sandwich shell. (a). $h_{c} / h=0.80$; (b) $h_{c} / h=0.95$. 

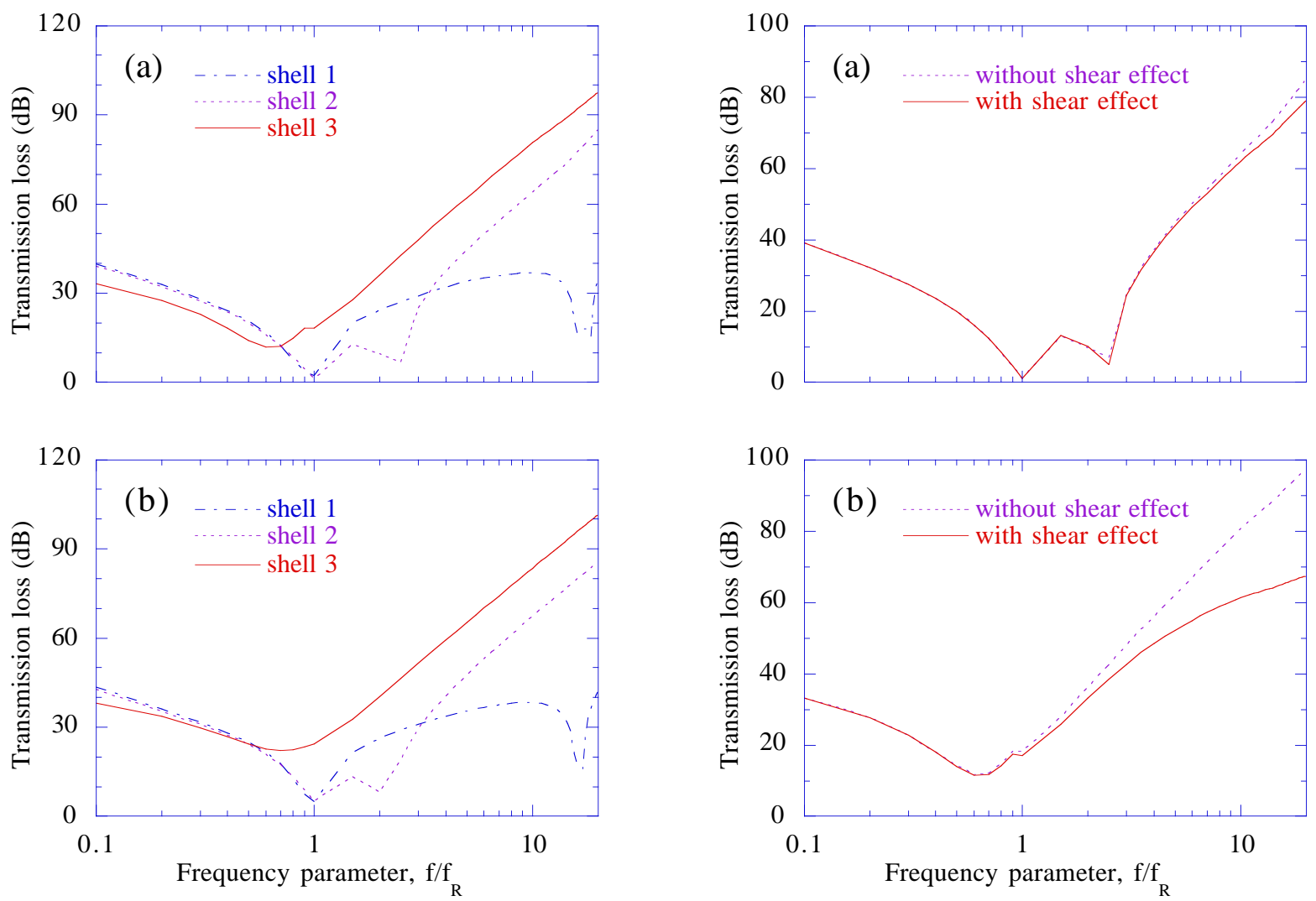

Figure 9. Comparison of the effect of the flight altitudes on the TL at no airflow with the interior pressure $(10,000 \mathrm{ft}): \eta=0.01 ; \gamma_{1}=150^{0} ; h_{c} / h=0.80$. shell 1: aluminum monocoque shell; shell 2: aluminum sandwich shell; shell 3: composite sandwich shell. (a). flight altitude 25,000 ft; (b) flight altitude 35,000 ft.

Figure 10. Comparison of the TL between the thin and the first-order deformation shell theories at no airflow with the flight altitude $(25,000 \mathrm{ft})$ and the interior pressure $(10,000 \mathrm{ft}): \quad \eta=0.01 ; \gamma_{1}=150^{0} ; h_{c} / h=0.8$. (a). aluminum sandwich shell; (b) composite sandwich shell. 